\title{
A Snapshot of Grassroots Primary Care and Tuberculosis Control in India-a US Fulbright-Nehru Scholar Program Experience
}

\author{
Sonal Shah Parikh, ${ }^{1}$ Juzar Ali, ${ }^{2}$ Sanjay Bhatt ${ }^{3}$ and DV Bala ${ }^{1}$
}

1. Community Medicine, Smt. NHL Medical College, Ahmedabad, Gujarat, India; 2. Louisiana State University Health Sciences Center (LSUHSC) School of Medicine, New Orleans, LA, US; 3. LOS Angeles County and University of Southern California (LAC+USC) Medical Center, Keck School of Medicine, LOS Angeles, CA, US

DOI: https://doi.org/10.17925/USRPD.2017.12.02.26

$\mathrm{T}$ he Department of Community Medicine, Smt. Nathiba Hargovandas Lakhmichand (NHL) Medical College, Ahmedabad, India, under the aegis of the US Fulbright-Nehru Scholar Program, reviewed the rural and urban primary and district health delivery system, with focus on primary care, child and maternal health and tuberculosis control. The National Health Mission program in India utilizes public and private resources and partnerships to optimally increase access, referrals and improve quality of care. As a collateral, the Revised National Tuberculosis Program, within its broader umbrella, incorporates digital-based locally applicable innovative approaches to tuberculosis (TB) control, to implement the World Health Organization strategy of the Directly Observed Treatment Short-course (DOTS) program. With this approach the program has consistently maintained a high treatment success rate. However, the deficiencies in the program include the loss to follow-up of missing persons with $\mathrm{TB}$, incomplete data in some cases, and erratic coordination between private, academic, public health primary and tertiary care centers.

\section{Keywords}

Primary care, National Health Mission (NHM), Revised National Tuberculosis Control Program (RNTCP), tuberculosis, US Fulbright-Nehru Scholar Program

Disclosure: Juzar Ali and Sanjay Bhatt were recipients of the Fulbright-Nehru International Scholar Award. Juzar Ali is also a member of the journal's editorial board. Sonal Shah Parikh and DV Bala have nothing to disclose in relation to this article. This article is an opinion piece and has not been submitted to external peer reviewers. Another member of the editorial board reviewed the article before publication.

Authorship: All named authors meet the International Committee of Medical Journal Editors (ICMJE) criteria for authorship of this manuscript, take responsibility for the integrity of the work as a whole, and have given final approval to the version to be published.

open Access: This article is published under the Creative Commons Attribution Noncommercial License, which permits any noncommercial use, distribution, adaptation, and reproduction provided the original author(s) and source are given appropriate credit.

Received: November 19, 2017

Published Online: December 12, 2017

Citation: US Respiratory \& Pulmonary Diseases, 2017;2(1):26-9

Corresponding Author: Sonal Shah Parikh, Community Medicine Department, Smt.

NHL Medical College, Ahmedabad, Gujarat, India.

E:dr.sonalparikh05@yahoo.com

Support: No funding was received in

the publication of this article.
India and the US, aside from being the world's two largest democracies with multi-ethnic communities and dynamic economies, face challenges in dealing with access to healthcare. Both countries have grappled with the demands of providing efficient and timely healthcare delivery, seeking to boost primary care and public health. It is clear that primary healthcare can play a critical role in preventing, detecting, and treating disease. Delivering primary care in India with a population of 1.3 billion, living in densely populated cities, rural areas and villages, and throughout many different sub-cultures, time zones, topographical domains, climates, languages, and dialects, is an enormous challenge. To address this challenge, public health officials and multi-level healthcare providers in Gujarat, India, as part of a national program, have implemented an innovative, grassroots program.

Juzar Ali and Sanjay Bhatt, as part of their Fulbright-Nehru International Scholar Award, along with a faculty team from Smt. Nathiba Hargovandas Lakhmichand (NHL) Medical College in Ahmedabad, Gujarat, India, explored and assessed this program.

The US Fulbright program was established in 1946 through the US Congress and was spearheaded by Senator JW Fulbright. It operates in 160 countries and has, to date, sponsored about 360,000 students, scholars, and senior faculty in all academic fields. It accepts reviews and approves applications and programs based on credentials, merit, needs assessment, communication, skills, and the commitment to further the goals of the Fulbright award. It expects its grantees to act as academic, teaching, and research scholars, and to be cultural ambassadors, to foster professional relationships and mutual goodwill (visit www.cies.org for further information).

\section{Background}

India has faced a significant void in achieving consistent, nationwide healthcare delivery, especially in three key categories: maternal and child health, immunization programs, and chronic communicable disease programs, including tuberculosis (TB) control. Despite many limitations, India does recognize the importance of primary healthcare and considers it to be the backbone of their health delivery system. Thus, inspired by the concept outlined by Sir Joseph Bhore in 1946, and three decades before the Alma Ata Declaration, emphasizing the importance of primary care, India has consistently worked to develop and streamline an effective approach to fill this void. Aided by the World Health Organization (WHO), the World Bank, UNICEF, and other international support agencies, the national 
and regional governments have put forth a concerted effort to develop a three-tier model for healthcare delivery. In April 2005, India launched the National Rural Health Mission (NRHM) which, alongside the National Urban Health Mission (NUHM), has revolutionized primary healthcare delivery. As part of this initiative, the primary healthcare system has adopted a proactive delivery model. Instead of delivering care strictly in hospitals, clinics, or offices, healthcare providers take care to the doorsteps of their patients. This is especially helpful in underserved and economically downtrodden urban slums and rural areas, where travel to and from healthcare facilities can be challenging. Under this primary level of care, irrespective of the socio-economic status of the family, every household in the rural community is regularly visited by healthcare providers. This is the first point of care and contact for individuals and families where basic essential utilitarian services are provided. The providers are based in local or regional sub-centers or primary health centers.

The secondary level of care is the community health center, the block-level primary health centers, and district hospitals. If a primary care provider detects medical needs requiring further assessment, these patients are referred to the secondary level of care. The final and tertiary level of care is provided by large public general hospitals, academic teaching institutions, and specialty clinics.

\section{Organizational structure}

Prior to the launch of the NRHM in 2005, all national health programs had vertical administrative and organizational structures. The hierarchy trickled down from the National Ministry of Health and Family Welfare to the district levels. With the launch of NRHM and NUHM, now called National Health Mission (NHM), all health programs are now covered under one convergent organizational umbrella. The NHM also offers financial flexibility between different health programs and utilizes all available resources optimally.1.2 Under less restrictive guidelines, expanded healthcare access, and streamlined referrals, the quality of care has improved. Furthermore, given the program's mission-driven rather than rules-based goals, public-private partnerships have been encouraged. Local program directors have the flexibility to establish partnerships with private institutions to encourage public health programs and campaigns. ${ }^{2}$ These relationships and escalating referral processes not only enable to focus on providing basic preventive healthcare, but help manage and maintain care in chronic public health diseases like TB. This part of the program was studied as outlined in the program case study below.

\section{Case study-tuberculosis}

Worldwide and especially in developing countries, TB is a rampant disease with devastating social and economic costs, and nowhere is that more evident than in India. More adults die in India from TB than from any other infectious disease. India has the world's highest global burden of both TB and multi-drug resistant TB (MDR-TB). Annually, an estimated 71,000 cases of MDR-TB are notified. Surveys from three of India's states indicate that 2.2\% of new TB cases and 15\% of previously-treated TB cases are MDR-TB (Tables 1 and 2). ${ }^{3.4}$ Further, based on estimates reported in the WHO Global Tuberculosis Report 2017, India has the second highest global burden of HIV-associated TB with an estimated 100,000 HIV-associated TB infections occurring annually, with a $31 \%$ mortality rate. ${ }^{3.4}$

The National Tuberculosis Control Program of India was initiated in 1962 and, using standard drug regimens for TB, was originally designed for
Table 1: Tuberculosis morbidity and mortality ${ }^{3,4}$

\begin{tabular}{llll}
\hline & Incidence & Mortality \\
\hline Global & $\begin{array}{l}10.40 \text { million } \\
140 \text { per } 100,000 / \text { year }\end{array}$ & 1.67 million \\
& 22 per 100,000/year \\
\hline \multirow{2}{*}{ India } & 2.79 million & 0.22 million \\
& 211 per $100,000 /$ year & 17 per $100,000 /$ year \\
\hline
\end{tabular}

Table 2: WHO estimated burden of tuberculosis in India ${ }^{3,4}$

\begin{tabular}{|l|l|l|}
\hline TB burden & $\begin{array}{l}\text { No. (millions) } \\
(\mathbf{9 5 \%} \mathrm{Cl})\end{array}$ & $\begin{array}{l}\text { Percentage } \\
(\mathbf{9 5 \%} \mathrm{Cl})\end{array}$ \\
\hline HIV among estimated incident TB patients & - & $\begin{array}{l}5 \% \\
(4.5-5.4 \%)\end{array}$ \\
\hline MDR-TB among pulmonary TB patients & 0.071 & - \\
\hline MDR-TB among new pulmonary TB patients & $(0.057-0.085)$ & 0.024 \\
\hline $\begin{array}{l}\text { MDR-TB among re-treatment pulmonary } \\
\text { TB patients }\end{array}$ & $0.021-0.029)$ & $\begin{array}{l}0.2 \% \\
(1.9-2.6 \%)\end{array}$ \\
\hline
\end{tabular}

$\mathrm{Cl}=$ confidence interval; $M D R=$ multi-drug resistant; $T B=$ tuberculosis; $W H O=$ World Health Organization

domiciliary self-administered treatment. ${ }^{4}$ The responsibility was placed squarely on the patient to seek out medical attention, follow-up care, and be compliant with the full treatment. Predictably, the results were mixed and fell far short of reducing the country's elevated level of disease.

With assistance from the World Bank, WHO, and other agencies, a Revised National Tuberculosis Control Program (RNTCP) was established in 1992. The key feature of this new plan was the Directly Observed Treatment Short-course (DOTS) strategy. From 1997-2006, the strategy was phased in and expanded nationally. Full nationwide coverage was achieved in March of 2006. Incorporating newer and more comprehensive approaches to TB control, and using modern methods of communication and engagement at all levels of society, the program has consistently maintained a high treatment success rate. Since 2007, the program boasts a treatment success rate greater than $85 \%$ and a new sputum positive case detection rate of $70 \% .5 .6$

\section{Key innovations of the program}

DOTS has thus become the backbone of RNTCP. The decentralized, community-based, drop-in primary health clinics have become the staple of most neighborhoods. In addition to their open-door, walkin policy, grassroots healthcare providers identify TB patients and guide them to the clinics. Patients can receive TB testing, diagnoses, information, education, drugs, follow up, and other care. The primary clinics act as a front-line of defense and an initial point of care for other illnesses as well. When any additional illness is identified, patients are also referred to the proper healthcare facility. Provider and patient incentives have helped reach even the most challenging areas. In some areas, this liaison occurs through volunteer help. Furthermore, modern digital technology is being incorporated to track patients in improved case-based surveillance systems. These include Nikshay, an innovative, successful, nationwide electronic TB registering and monitoring program. The "99DOTS" strategy is a web-based monitoring tool developed to 
keep track of TB patients and ensure quality case management. It is a low-cost approach for monitoring and improving TB medication adherence by using mobile phones and text messaging at no cost to the patients, through a locally used common process of "missed calls". After taking their daily medications, patients make a mandatory, but free, call to the phone number code-wrapped in each anti-TB medicine blister pack. This in turn notifies that each patient is correctly taking their medicine on schedule. If a patient phone call is not received, it is identified as a possible deviation from the accepted treatment protocol. These deviations are registered, documented, and can be promptly addressed by healthcare workers who contact the patient personally through their health units. By some accounts, the "99DOTs" program has improved medication adherence by over $90 \%$. Another critical aspect of the program's success has been careful attention to the treatment of children with TB. Parents and family members are trained to administer care, and child-friendly fixed dosage combinations.

A "Tribal Action Plan" has been developed to improve access to specific ethnic and other marginalized groups. This plan creates additional TB units and designated microscopy centers (DMCS). DMCs are in remote locations, equipped with additional staff at a relatively higher salary, who provide compensation for the transportation of the patient and attendant.7.8 Public-private mix (PPM) schemes have been formulated to encourage non-governmental organizations (NGOS) and private practitioners to participate and contribute to TB control efforts. ${ }^{8}$ These efforts are supported by international (including US-based) private foundations and organizations.

One of the most successful of these PPM schemes has been the combined effort to provide patients in the private sector with free medications. When a patient visits a primary care physician and is diagnosed with TB, they are eligible for free medications rather than being referred to a new medical system. In 2014, the Ministry of Health and Family Welfare started a pilot project in Mumbai to provide free treatment to private patients. This project, called the Private Practitioner Agency (PPA), has proven largely popular and successful. Under this program, a TB patient goes to a PPAregistered facility where physicians issue vouchers for the required $\mathrm{X}$-rays and medications. The government reimburses pharmacies, diagnostic centers, and laboratories for these services. The patient's only cost is the private physician's consultation fee. ${ }^{9}$

TB is predominantly diagnosed in India using smear microscopy. It was observed that sputum collection and culture is not routinely performed in newlydiagnosedcases. Quality assurance for the sputumsmearmicroscopy is implemented through a three-tier system consisting of national reference laboratories, intermediate reference laboratories, and DMCs. Cartridge-based nucleic acid amplification sites provide rapid decentralized diagnosis of MDR-TB, as well as TB in HIV-positive and in pediatric patients.

\section{Results}

Through these innovative efforts to enhance the implementation of the internationally recognized DOTS program, India has made considerable progress in controlling TB. It has achieved the United Nations TB Millennium Development Goals and the WHO STOP TB targets of 50\% reduction in TB prevalence and mortality, ${ }^{4} 58 \%$ reduction in TB mortality rate, and 55\% reduction in TB prevalence rate by 2014 (as compared to 1990 levels). The incidence of TB has been reduced from 216 per 100,000 in 1990 to 167 per 100,000 in 2014. Of all registered TB cases, 79\% knew their HIV status; 93\% of HIV-infected TB patients were initiated on chemoprophylaxis treatment, and $92 \%$ were initiated on anti-retroviral therapy. Re-treatment success rate after relapse and default is $75 \%$ and $68 \%$, respectively. ${ }^{5}$

The RNTCP has been successful, and many other programs have partnered with its successful approach. To fight against HIV-associated TB, the National AIDS Control Program (NACP) and RNTCP have jointly developed a "national framework of joint TB/HIV collaborative activities".? Under this program, all suspected cases found positive for TB are also screened for HIV.

Additionally, RNTCP and the National Program for Prevention and Control of Cancer, Diabetes, Cardiovascular Disease \& Stroke (NPCDCS) have jointly developed a framework for collaboration which aims to reduce morbidity and mortality by performing bi-directional screening, early detection, and prompt management of diabetes mellitus and TB. This combination of co-morbidities has now been recognized by WHO and other world health bodies as needing immediate attention. NTCP and the National Tobacco Control Program are also synergistically working to develop and implement a framework for collaboration. ${ }^{5}$

\section{Challenges}

Although the program is widely viewed as a public health success, significant gaps and challenges remain. These include the following.

- Drug-resistant TB: while the treatment success rate is $46 \%$, it carries a $20 \%$ mortality and $20 \%$ of patients are lost to follow-up. ${ }^{10}$ Resistance to first-line drugs, random use of fluoroquinolones, lower body mass index, and history of previous incomplete or erratic treatment account for some of these cases. Non availability of results of previous culture and sensitivity of sputum if at all performed, and limited follow-up, noticeably in urban areas add to this public health challenge.

- The right to refuse treatment: under the Human Rights Act, a patient may refuse care or treatment, even in cases of highly infectious disease, such as TB. A patient's rights take precedence over the rights of society at large. ${ }^{11}$ There is no legal provision to compel patients to complete treatment or quarantine them. Even after extended counselling, healthcare providers are helpless if a patient wishes to be non-compliant with further treatment. Such cases remain a continuous source of contagion and a hidden reservoir of infection.

- Private practitioners: undoubtedly these practitioners serve an invaluable purpose in the delivery of healthcare to the public. Private healthcare providers provide most of the overall access to care. Ironically though, they are also part of the problem. Urban areas tend to have weaker and more inconsistent primary healthcare systems than their rural counterparts, based on individual area variation and mobility of patients with "physician shopping". Thus, TB cases may be initially managed differently, national guidelines may not necessarily be followed, and initial cost of treatment may not be affordable for patients. Throughout India, at least half of TB patients, and $80 \%$ of the first contact of the patients from all socio-economic groups, seek initial care through private healthcare providers. This includes qualified and unqualified practitioners, alternate medicine care providers, pharmacies, and laboratories. Patient and family 
preferences and biases add to the mix of this complex public health amalgam. Incomplete notification of cases, irrational anti-TB drug use, and poor patient follow-up are some of the other important barriers towards achieving comprehensive consistent TB control. Nearly 50\% of the re-treatment cases notified under RNTCP are treated in private sector or non-structured systems before reaching RNTCP, suggesting inadequate treatment and possible amplification of drug resistance (unpublished data from Central TB Division, Government of India). This remains one of RNTCP'S key challenges. Existing strategies are constantly being redesigned to counter this challenge under RNTCP's National Strategic Plan. ${ }^{8}$ India is now responsible for nearly onethird of the world's "missing TB cases" believed to be managed by private practitioners. ${ }^{3}$ The private health sector has unfortunately been out of the loop, thus perpetuating poor notification, and in some ways enabling fragmentation of treatment plans, leading to increased chances of drug resistance. RNTCP has made concerted efforts to engage the private sector in India. 12,13
- Coordination of care: the challenge of streamlining the coordination of care between private, academic, tertiary, and public health sectors; lack of synergism of conventional and non-conventional medicine; disengaged providers of alternative medicine; and lack of liaison between private care and national registries, remains a daunting task. Failure in this aspect is a crucial factor in achieving TB control, reducing missed cases, and avoiding the emergence and persistence of drugresistant cases of TB in India.

\section{Conclusion}

India's approach of using innovative, patient-centered locally placed measures to implement TB control demonstrates areas of documented success. This is despite continued challenges of high demand, limited capacity and available resources. The proven concept of the DOTS program notwithstanding, the importance of "connecting the dots" between primary care, TB control programs and private care, with robust coordination strategies and health information exchange, cannot be overemphasized. $\square$
1. Ministry of Health and Family Welfare, Government of India, National rural health mission document, New Delhi, India: 2005-2012, Available at: www.pbnrhm.org/docs/mission_doc.pd (accessed December 3, 2017).

2. Ministry of Health and Family Welfare, Government of India National rural health mission: meeting the people's health needs in partnership with states, the journey so far, New Delhi, India: 2005-2010, Available at: https://nrhm-mis.nic.in/NRHM\%20 Publications/Five\%20Years\%20of\%20NHM\%202005-2010.pdf (accessed December 3, 2017)

3. Central TB Division, Ministry of Health \& Family Welfare, Government of India, Technical and operational guidelines for TB control in India 2016, Available at: https://tbcindia.gov.in/ index1.php?lang=1\&level=2\&sublinkid=4573\&lid=3177 (accessed November 20, 2017)

4. World Health Organisation (WHO), Global Tuberculosis Report 2017, Geneva, Switzerland: 2017, Available at: http://apps.who. int/iris/bitstream/10665/259366/1/9789241565516-eng.pdf? (accessed December 3, 2017)
5. Sachdeva KS, Kumar A, Dewan P, et al., New vision for the revised national tuberculosis control programme (RNTCP): universal access - "reaching the un-reached", Indian J Med Res, $2012: 690-4$

6. Central TB Division, Ministry of Health and Family Welfare, Government of India, TB India 2010. RNTCP status report, Available at: https://tbcindia.gov.in/showfile.php?lid=2922 (accessed December 3, 2017)

7. Central TB Division, Ministry of Health and Family Welfare Government of India, Tribal action plan (proposed for the world bank assisted RNTCP II project), New Delhi, India: April 2005, Available at: http://documents.worldbank.org/curated/ en/603531468243865797/pdf/IPP127.pdf (accessed December 3 , 2017).

8. Central TB Division and WHO country office for India, Universal access to TB care (UATBC), concurrent assessment report, New Delhi, India: May 2016, Available at: www.searo.who.int/india/ publications/universal_access_to_tb_care_part1.pdf (accessed December 3, 2017).
9. Global Health Education (GHE), TB in India - Elimination, private care, TB burden, NSPS. Available at: www.tbfacts.org/tb-india accessed November 20, 2017).

10. Central TB Division, Ministry of Health \& Family Welfare, Government of India, Revised National Tuberculosis Control Programme, National strategic plan for tuberculosis control 2012-2017, Available at: https://www.tbfacts.org/wp-content/ uploads/2016/01/NSP-2012-2017.pdf (accessed November 20, 2017)

11. Parikh $\mathrm{K}$, The right to refuse treatment, Indian J of Med Ethics, 2003:11:3.

12. Central TB Division, Ministry of Health \& Family Welfare, Government of India, TB India 2016, Revised National TB Control Programme Annual Status Report, Available at: https://tbcindia.gov.in/showfile.php?lid=3180 (accessed November 20, 2017)

13. Pai M, Formidable killer: drug-resistant tuberculosis, The Tribune, India, August 6, 2013, Available at: http://www.tribuneindia. com/2013/20130806/edit.htm\#6 (accessed November 20, 2017). 